\title{
Correction to: The old CEACAMs find their new role in tumor immunotherapy
}

\author{
Zi-Wen Han ${ }^{1,2} \cdot$ Zhi-Wu Lyv $^{3} \cdot$ Bin Cui ${ }^{3} \cdot$ Ying-Ying Wang ${ }^{1,2} \cdot$ Jun-Ting Cheng ${ }^{1,2} \cdot$ Ying Zhang $^{1,2} \cdot$ Wen-Qi Cai ${ }^{1,2}$. \\ Yang Zhou ${ }^{1,2} \cdot$ Zhao-Wu Ma $^{1,2}$ - Xian-Wang Wang ${ }^{1,2,4} \cdot$ Xiao-Chun Peng ${ }^{1,2,5}$. Shu-Zhong Cui ${ }^{6}$ - Ying Xiang ${ }^{1,2}$. \\ Mo Yang ${ }^{7} \cdot$ Hong-Wu Xin ${ }^{1,2}$
}

Published online: 22 June 2020

(C) Springer Science+Business Media, LLC, part of Springer Nature 2020

\section{Correction to: Invest New Drugs https://doi.org/10.1007/s10637-020-00955-w}

Correction is needed to the original version of this article. The extra repeated "Urinary Testicles" and the corresponding bar on the horizontal axis in Fig. 2 should be removed.

The authors apologize for the error and further state that the correction made to the image does not affect the overall outcome of the study. The corrected figure is shown below.

Publisher's note Springer Nature remains neutral with regard to jurisdictional claims in published maps and institutional affiliations.

The online version of the original article can be found at https://doi.org/ 10.1007/s10637-020-00955-w

Ying Xiang

xying316@163.com

$\triangle$ Mo Yang

Yangm1091@126.com

$\triangle$ Hong-Wu Xin

hongwu_xin@126.com

1 Laboratory of Oncology, Center for Molecular Medicine, School of Basic Medicine, Health Science Center, Yangtze University, 1 Nanhuan Road, Jingzhou 434023, Hubei, China

2 Department of Biochemistry andMolecular Biology, School of Basic Medicine, Health Science Center, Yangtze University,

Jingzhou 434023, Hubei, China
3 Department of Pathology, Lianjiang People's Hospital, Zhanjiang 524400, Guangdong, China

4 Department of Laboratory Medicine, School of Basic Medicine, Health Science Center, Yangtze University, 1 Nanhuan Road, Jingzhou 434023, Hubei, China

5 Department of Pathophysiology, School of Basic Medicine, Health Science Center, Yangtze University, Jingzhou 434023, Hubei, China

6 State Key Laboratory of Respiratory Disease, Affiliated Cancer Hospital Institute of Guangzhou Medical University, Guangzhou 510095, China

7 The Seventh Affiliated Hospital Sun Yat-sen University, Shenzhen, 11, Guangdong Province 518107, People's Republic of China 


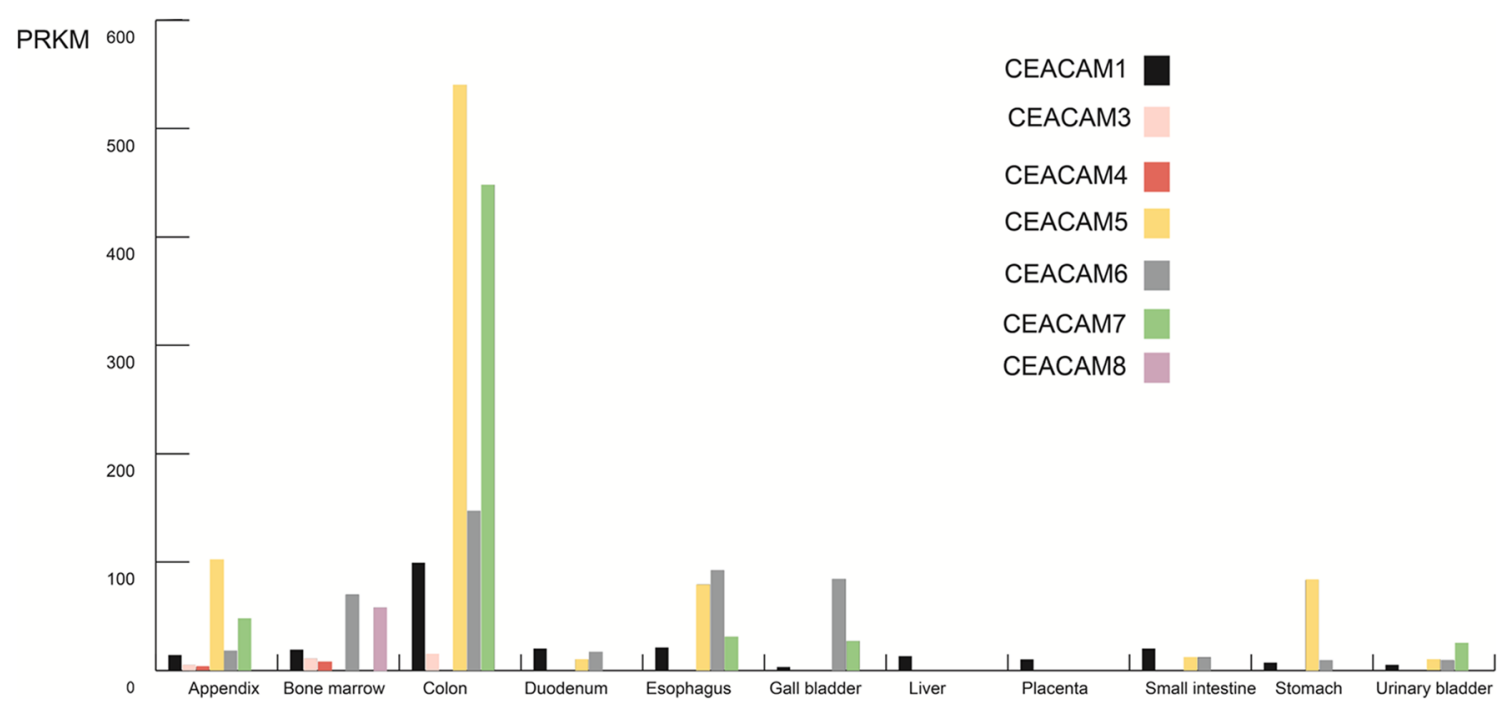

Fig. 2 The expression of CEACAMs in normal tissues. The expression of CEACAM1, CEACAM3, CEACAM4, CEACAM5, CEACAM6, marrow, colon, duodenum, esophagus, gall bladder and liver was shown. PRKM: Reads Per Kilobase of exon model per Million mapped reads CEACAM7 and CEACAM8 in normal tissues, including appendix, bone 